\title{
Rainbow Plasmonic Nanobubbles: Synergistic Activation of Gold Nanoparticle Clusters
}

\author{
Ekaterina Y Lukianova-Hleb ${ }^{1}$, Alexander O Oginsky ${ }^{1,2}$, Derek L Shenefelt ${ }^{3}$, Rebekah A Drezek ${ }^{4}$, Jason H Hafner ${ }^{5}$, Mary C Farach-Carson ${ }^{3}$ and Dmitri O
} Lapotko ${ }^{1,5 *}$

1 Joint American-Belarusian Laboratory for Fundamental and Biomedical Nanophotonics, Rice University, 6100 Main Street, Houston, TX, 77005, USA

${ }^{2}$ Belarusian State University for Informatics and Radioelectronics, 6 Browka Street, Minsk, 220013, Belarus

${ }^{3}$ Department of Biochemistry \& Cell Biology, Rice University, 6100 Main Street, Houston, TX, 77251-1892, USA

${ }^{4}$ Department of Bioengineering, Rice University, 6100 Main Street, Houston, TX, 77251-1892, USA

${ }^{5}$ Department of Physics \& Astronomy, Rice University, 6100 Main Street, Houston, TX 77005, USA

\begin{abstract}
The synergistic physical and biological effects of selective targeting and activation of plasmonic nanoparticles were studied for a transient vapor nanobubble mode. Simultaneous optical activation of two plasmon resonances in multinanoparticle clusters significantly improved the selectivity and efficacy of the nanobubble generation through and was termed "rainbow plasmonic nanobubbles." The rainbow nanobubble mechanism has been studied in water and in living cells in vitro. This mechanism provided maximal selectivity of the nanobubble generation in both models and therefore, can the therapeutic selectivity and optical contrast of gold nanoparticles in a heterogeneous physiological microenvironment at cell level.
\end{abstract}

Keywords: Plasmonic nanobubble; Gold nanoparticle; Laser; Photothermal; Cell; Cancer

\section{Introduction}

The biomedical applications of plasmonic nanoparticles (NPs) use their optical scattering properties for imaging and diagnostics [1-4], and their photothermal properties for various types of therapies through the generation of heat $[1,5,6]$, vapor bubbles [6-12] and acoustic waves $[13,14]$. The specificity and sensitivity of imaging and diagnostics as well as the selectivity and efficacy of the therapeutic methods depend upon the selectivity of NP delivery and activation within their targets, typically diseased cells or tissues. The initial application of various NPs was based on passive targeting [15-17], which did not allow the NPs to recognize their specific molecular targets and resulted in the low selectivity of NP-based methods and also in potentially toxic loads of NPs. The situation was improved by using active molecular targeting with cell-specific molecules (peptides, antibodies, aptamers) attached to NPs and coupling to cognate receptors at the membranes of specific target (diseased) cells [18-21]. This approach is currently used for the in vitro and in vivo application of NP-based imaging, diagnostics and therapies including drug delivery with gold NPs [22-26]. However, it still does not provide "absolute" targeting: certain amounts of NPs are taken up nonspecifically by normal cells and tissues instead of diseased cells. Consequently, the specificity and sensitivity of imaging and diagnostics, and the selectivity and efficacy of the therapeutic methods, are reduced. On the other hand, the major promise of NPbased medicine is associated with the unique properties of NPs and the nanoscale processes they support. To realize this promise, one needs a targeting mechanism that provides selective delivery and activation of the NPs only in target cells without influencing other adjacent normal cells. Otherwise NP-based medicine often defaults to its "macro" version by employing only the bulk effects of NPs, and thus losing their main advantage over other materials and methods.

To address this problem, we recently introduced the antibodyendocytosis based mechanism of gold NP targeting and activation through generating transient vapor bubbles (plasmonic nanobubbles) [27-29]. This mechanism used the selective formation of NP clusters (tightly aggregated groups of 5-50 NPs) in specifically targeted cells and the cluster size-dependent mechanism of optical activation of such clusters [28-32]. The two key processes employed were endocytosis (big NP clusters were formed only in diagnosis-specific cells, while fewer NPs incidentally accumulated by non-specific cells were insufficient to form an NP cluster as big as in the specific cells) and plasmonic nanobubbles (PNBs), transient vapor bubbles generated around plasmonic NP clusters under their exposure to a short laser pulses. PNB provided dual tunable mechanical and optical action at cell level [27, 31, 33]. Unlike many other thermal, acoustical and optical phenomena related to NPs and lasers, the generation of PNBs is a threshold event: PNB does not emerge if the laser pulse energy is below specific threshold. The PNB threshold energy can be significantly decreased with the NP cluster size [30-32], thus allowing the selective generation of PNBs only around big enough clusters that cannot be found in non-specific cells. The dynamically tunable optical and mechanical properties of PNBs make them ideal candidates for cell level imaging, diagnostics, therapy and theranostics $[27,31,33]$.

However, a general heterogeneity of biological properties (including the expression of specific receptors and their ability to support endocytosis in non-specific cells) often reduces the selectivity of NP targeting and the precision of the following diagnosis and therapy [21,34-36]. As results, NP-based biomedical technologies often cannot provide real "nano" targeting at cell level. We hypothesized that a further improvement in the selectivity of NP-based imaging, diagnosis and therapy could be achieved through the simultaneous activation of the several different and colocolized plasmon resonances in one NP

*Corresponding author: Dmitri O Lapotko, Rice University, MS 61, 6100 Main Street, Houston, TX 77005, Tel: 713-348-3708; Fax: 713-348-4150; E-mail: d15@ rice.edu

Received November 04, 2010; Accepted January 18, 2011; Published January 21,2011

Citation: Lukianova-Hleb EY, Oginsky AO, Shenefelt DL, Drezek RA, Hafner $\mathrm{JH}$, et al. (2011) Rainbow Plasmonic Nanobubbles: Synergistic Activation of Gold Nanoparticle Clusters. J Nanomedic Nanotechnol 2:104. doi:10.4172/21577439.1000104

Copyright: (c) 2011 Lukianova-Hleb EY, et al. This is an open-access article distributed under the terms of the Creative Commons Attribution License, which permits unrestricted use, distribution, and reproduction in any medium, provided the original author and source are credited. 
cluster in order to generate plasmonic nanobubbles in more selective way. Our current work was focused on the experimental evaluation of this hypothesis for PNBs generated in water and in living cells.

\section{Materials and Methods}

\section{Generation of rainbow plasmonic nanobubbles}

The generation of the vapor bubble around a plasmonic NP (Figure 1A) requires that the fluence of the excitation (pump) laser pulse exceeds a specific threshold. When the threshold is exceeded, the NP delivers a sufficient amount of thermal energy to the NP nano-environment, enables its rapid evaporation and the consequent expansion of the vapor into the nanobubble (PNB). The clustering of plasmonic NPs was shown to reduce the PNB generation threshold [30-31]. The described mechanism employs one plasmon resonance (one type of plasmonic NPs) as the source of heat. We expanded this approach into a principally new mechanism that employs the simultaneous effect of several different and colocalized plasmon resonances in one NP cluster. This was realized through using two different types of plasmonic (gold) NPs, nanorods (near-infrared plasmon resonance) and nanospheres (visible plasmon resonance), and their mixed clusterization (Figure 1B). If this multi-NP cluster is irradiated with one laser pulse at subthreshold fluence (below the PNB threshold for clusters of a similar size though built of only one type of NP) there will be no bubble (Figure 1C). However, if we irradiate this multi-NP cluster with the two simultaneous pulses at two different wavelengths (matching the plasmon resonances of NPs), their cumulative thermal effect will exceed the PNB threshold and will result in a PNB (Figure 1C). This mechanism was termed as "rainbow plasmonic nanobubble" because it employs several different wavelengths of laser radiation and this mechanism is principally different from the standard one:

1. A single source of optical energy (a laser pulse at a single wavelength) is replaced by several sources (several simultaneous pulses of different wavelengths), while the fluence of each pulse is reduced below the PNB generation threshold for each corresponding NP type. In such cases, the simultaneous exposure of the mono-NP cluster or the single pulse exposure of the mono- or multi-NP cluster will not generate a PNB, since the thermal output in both cases will be insufficient, and only the simultaneous excitation of the multi-NP cluster (rainbow mechanism) will cause the bubble.

2. A reduced fluence of laser pulses results in a lower initial temperature of individual NPs in a multi-NP cluster compared to the case of a single pulse excitation of a mono-NP cluster. The simultaneous heating of closely packed different NPs in the cluster results in a rapid fusion (superposition) of their thermal fields into one joint thermal field surrounding the NP cluster. This secondary thermal field acts as the energy source for the vapor bubble formation and expansion, and, compared to the single plasmon resonance case, the thermal load on the NPs is reduced, thus better protecting them from melting and enabling their multiple activation.

3. The independent control of the fluence of each laser pulse allows us to optimize the excitation of the multi-NP cluster for maximal selectivity of PNB generation, whereas excitation with a single pulse does not offer this opportunity.

From the biomedical point of view the above features can be considered as a reduction of the radiation load on a tissue and an improvement in PNB selectivity in a heterogeneous cell environment when different NPs are targeted with the different vectors (antibodies, peptides etc). Optical generation of the PNBs was performed with a previously developed photothermal laser microscope [37] equipped with a dual pulse laser (STH-01, Standa Ltd, Vilnius, Lithuania): each $0.5 \mathrm{~ns}$, the wavelength $532 \mathrm{~nm}$ (matching plasmon resonances of gold spheres of $60 \mathrm{~nm}$ ) and 787nm (matching plasmon resonances of gold nanorods and nanoshells that were employed). The pump laser beams were directed into the illumination path of an inverted optical microscope and were focused into the sample. Single cells or individual NP clusters and NPs and single events were studied. The pump beam diameter was measured at the level of half of the maximum in order to estimate the maximal level of the excitation fluence (standard definition of the fluence employs the beam diameter measured at the level of $1 / \mathrm{e}^{2}$ relative to the maximal intensity in the center of the beam).

\section{Optical detection of plasmonic nanobubbles and gold nanoparticles}

Optical detection of PNBs was realized in two parallel modes: time-resolved scattering imaging (Figure 2A) and time response (Figure 2C). Time-resolved scattering imaging was realized by using side illumination of the sample with a pulsed probe laser beam at a wavelength $690 \mathrm{~nm}$, different from the pump laser wavelengths (532 $\mathrm{nm}$ and $787 \mathrm{~nm}$ ). Optical scattering was also used for the imaging of single NPs and their clusters. For the objects whose dimensions are smaller than a wavelength the size of their image does not represent their actual size. However, if the image is formed by the light scattered by the object, the brightness of the scattered light (measured as the pixel image amplitude) correlates to the size of the object. We have used this well known rule for quantitative control of the relative size of the gold nanoparticle clusters. This provided the comparison of the clusters of similar size. The scattered by NP or by PNB probe radiation was imaged with the CCD camera (Luka, Andor Technologies, Belfast, Northern Ireland). While allowing to "see" the PNB or NP a time-resolved scattering imaging cannot provide kinetic measurement. The latter was

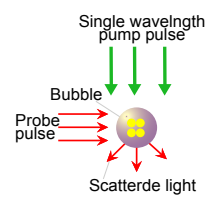

A
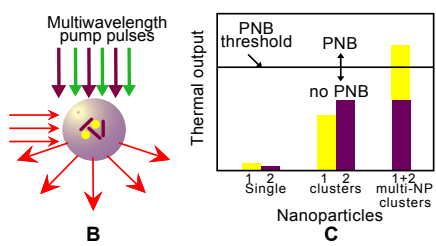

C
Figure 1: Principle of optical generation and detection of: $(A)$ a standard PNB induced with a single laser pulse around a mono-NP cluster, and (B) a rainbow PNB induced with several simultaneous laser pulses (shown with green and purple arrows) and a multi-NP cluster (yellow and purple NPs with different plasmon resonances); (C) thermal energy released by a single NP, mono-NP clusters and multi-NP cluster under identical optical excitation with two laser pulses, like in case (B): only simultaneous excitation of two plasmon resonances in one multi-NP cluster delivers thermal energy sufficient for a PNB. Red arrows in (A) and (B) show PNB detection through optical scattering of an additional probe laser beam.
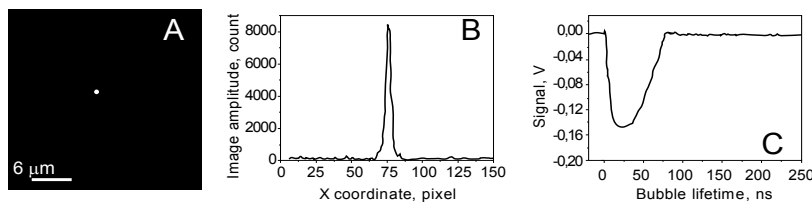

Figure 2: (A) Time-resolved scattering optical image shows individual transient PNB generated around NP cluster exposed to the two simultaneous laser pulses $(0.5 \mathrm{~ns}$ each, $532 \mathrm{~nm}$ and $787 \mathrm{~nm})$; (B) - the profile of pixel image amplitude for the image shown in $A$, and $(C)$ : corresponding time response (obtained for the same PNB simultaneously with the image A) shows PNB specific dip-shaped signal and characterizes maximal diameter of the PNB. 

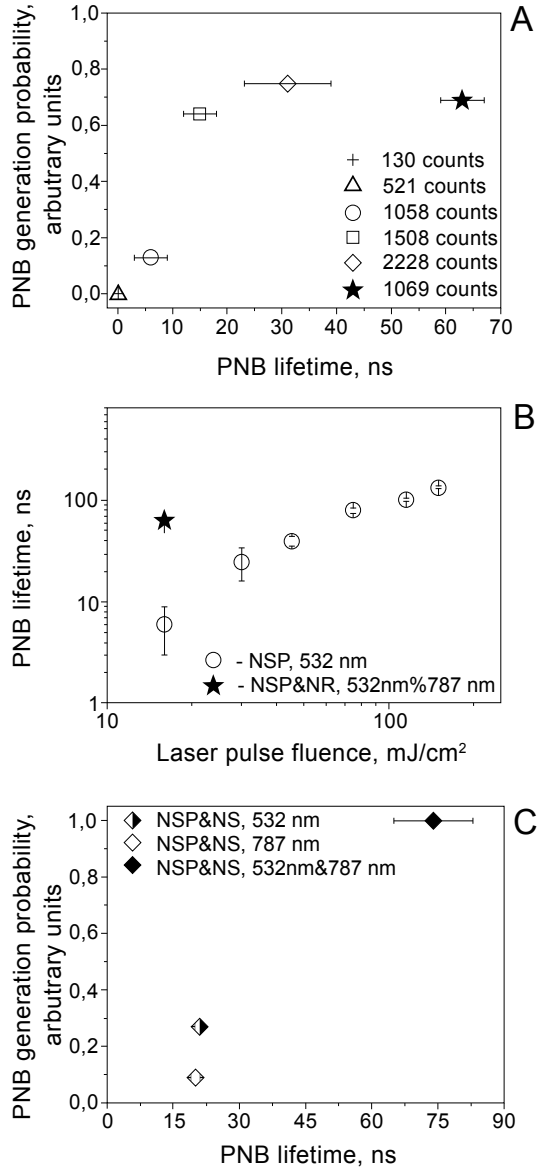

Figure 3: (A) Lifetime and probability of PNBs generated around clusters of 60 nm gold NSPs in water and measured as functions of the NP cluster size (single 0.5 -ns laser pulse $\left.532 \mathrm{~nm} @ 16 \mathrm{~mJ} / \mathrm{cm}^{2}\right)$, solid star - rainbow PNB generated around multi-NP clusters of NSPs (plasmon peak close to $532 \mathrm{~nm}$ ) and NRs (plasmon peak close to $787 \mathrm{~nm}$ ) with two simultaneous 0.5-ns laser pulses (532 nm@16 $\mathrm{mJ} / \mathrm{cm}^{2}$ and $787 \mathrm{~nm} @ 36 \mathrm{~mJ} / \mathrm{cm}^{2}$, the rainbow mechanism); (B) lifetime of PNBs as a function of the laser pulse $(532 \mathrm{~nm})$ fluence for mono-NP lifetime of PNBs as a function of the laser pulse $(532 \mathrm{~nm}$ ) fluence for mono-NP
clusters of NSPs (star symbol shows the rainbow PNB generated around a multi-NP cluster of NSPs and NRs with two simultaneous $0.5 \mathrm{~ns}$ laser pulses (532 $\mathrm{nm} @ 16 \mathrm{~mJ} / \mathrm{cm}^{2}$ and $787 \mathrm{~nm} @ 36 \mathrm{~mJ} / \mathrm{cm}^{2}$, the rainbow mechanism); (C): PNB lifetime-probability diagram for multi-NP clusters of mixed NSPs and NSs under identical excitation with one $0.5 \mathrm{~ns}$ laser pulse $532 \mathrm{~nm} @ 40 \mathrm{~mJ} / \mathrm{cm}^{2}$, one pulse $787 \mathrm{~nm} @ 48 \mathrm{~mJ} / \mathrm{cm}^{2}$, and two simultaneous laser pulses - $532 \mathrm{~nm}$ and $787 \mathrm{~nm}$ (the rainbow mechanism).

realized with a simultaneous time response mode [37]. An additional continuous probing beam $(633 \mathrm{~nm})$ was directed to the sample and focused on it collinearly with pump laser beams and its axial intensity was monitored by a high-speed photodetector (PDA110AC, Thorlabs Inc., Newton, NJ) and 400-MHz LeCroy digital oscilloscope (42XsA, LeCroy Corporation, Chestnut Ridge, NY). The PNB-induced scattering of a part of the probe beam decreased its axial amplitude resulting in a dip-shaped output signal [37]. This mode provided the monitoring of a PNB growth and collapse and delivered the PNB lifetime that characterizes its maximal diameter [37]. The PNBs were quantified by measuring their lifetime (Figure 2C), bubble generation threshold fluence and the pixel amplitude of their scattering images (Figure 2B). The PNB generation threshold fluence of the pump laser pulse describes both the efficacy of the photothermal conversion by NP and hydrodynamic conditions of the PNB generation. It was defined as pump laser pulse fluence that provides PNBs generation probability 0.5 .

\section{Experimental models}

NP clusters in water: Three types of gold NPs were studied: gold spheres (NSP) (60 nm diameter), gold rods (NR) $(25 \mathrm{~nm} \times 75 \mathrm{~nm})$ and $110 \mathrm{~nm}$ gold shells (NS) with silica core inside. NSPs and NRs were obtained from Nanopartz (Salt Lake City, UT). Gold shells (NS) were prepared by us. NP clusters were prepared by adding PBS into the suspension of single NPs (1:1). After $20 \mathrm{~min}$ the NP clusters were resuspended in water in order to adjust their concentration. Three types of the clusters were studied: mono-NP clusters of NSP, mono-clusters on NR, and the multi-NP (NSP-NR) cluster consisting of the NSPs and NRs. Another set of mono- and multi-NP clusters was prepared with the NSPs and NSs. Optical scattering amplitudes of the NP cluster images were used to pick only identical clusters so to avoid the influence of the variation of their diameters. Formation of the clusters has been verified with the two methods: by scanning electron microscope (SEM) (FEI Quanta 400 ESEM FEG), and optical scattering microscopy. We have used optical scattering amplitude (measured with CCD and with the LSM-710 laser scanning microscope (Carl Zeiss MicroImaging $\mathrm{GmbH}$, Germany) as the relative measure of the cluster size: only the objects with the close optical scattering amplitudes were used for the experiments. Based on their SEM images, the size of these clusters in each sample was 130-180 nm. Water samples of gold NP clusters were prepared on standard microscope slides and circle coverslips. The individual NP clusters were positioned into the center of laser beams and exposed to the single or paired laser pulses.

Living cell model: The selectivity and sensitivity of plasmonic nanobubbles as cellular probes were studied in a co-culture of $\mathrm{C} 4-2 \mathrm{~B}$ prostate cancer cells and non-cancer stromal HS-5 cells. C4-2B cells were selectively targeted with gold NPs using two cancer-specific surface markers: anti-prostate membrane antigen (PSMA) that is primarily expressed in prostatic tissues as well as in bone metastases $[38,39]$ (PSMA enzymatic activity is high in C4-2B cells [40]) and C225 (anti-EGFR) that is expressed in prostate cancer and malignancies [41]. C4-2B prostate cancer cells and HS- 5 human bone stromal cells were maintained either alone or in co-culture as described previously [42]. Cells were grown to $80 \%$ confluency and then were treated with $60 \mathrm{~nm}$ gold NSPs conjugated to PSMA and $110 \mathrm{~nm}$ gold NSs conjugated to $\mathrm{C} 225$ antibodies for $30 \mathrm{~min}$ at $37^{\circ} \mathrm{C}$. The formation PSMA and C225 antibody-receptor complex induces uptake of nanoparticles through endocytosis resulting in nanoparticle clusters $[43,44]$. The accumulation of gold NPs in living cells was directly monitored with optical scattering imaging performed with a laser scanning confocal microscope LSM710 (Carl Zeiss MicroImaging GmbH, Germany) in the scattering mode under excitation with a $633 \mathrm{~nm}$ continuous laser.

\section{Results and Discussion}

\section{Rainbow plasmonic nanobubbles in water}

Initially we studied the generation of PNBs around mono-NP and multi-NP clusters in water. Imaging of the PNBs was realized in two parallel modes: time-resolved scattering imaging showed individual transient PNBs at the moment of their generation (Figure 2A), and time-response mode (Figure 2C) employed a continuous probe laser $(633 \mathrm{~nm})$ to monitor the PNB growth and collapse and thus to obtain the PNB lifetime that characterizes the maximal diameter of the PNB [37]. Mono-NP clusters consisting of the NSPs were analyzed the first for the influence of the cluster size on the maximal diameter (the lifetime) and generation probability of PNB (Figure 3A). The size of $\mathrm{NP}$ clusters was quantified and compared through the amplitudes of their optical scattering images (obtained for individual NP clusters). In 
scattering imaging of the object with sub-wavelength size, their image size does not correspond to their real size. However, the amplitude of the scattering image amplitude strongly depends upon the object diameter (the sixth power): the bigger the NP cluster, the higher the image scattering amplitude. At the fixed fluence of $16 \mathrm{~mJ} / \mathrm{cm}^{2} @ 532$ $\mathrm{nm}$ (the wavelength of plasmon resonance of NSPs), the single NPs and their small mono-NP clusters did not generate PNBs (Figure 3A). The increase of the NP cluster size (measured as optical scattering amplitude at Figure 3A) allowed us to overcome the PNB generation threshold and the PNB lifetime was found to be nearly proportional to the size of the NP cluster (Figure 3A). In fact, the increase of the cluster size influenced both the PNB generation probability and the lifetime (Figure 3A). Therefore, under the level of optical excitation fluence set below the PNBs threshold for single NPs or their small clusters, the bigger NP clusters supported the generation of PNBs. For the NP cluster of fixed size (we have considered the NP clusters with the level of scattering amplitude around 1060 counts) the increase of the optical excitation fluence above the PNB generation threshold produced bigger PNBs (Figure 3B) while the dependence of the PNB maximal diameter (lifetime) upon fluence level was close to linear.

Next we compared the PNBs generated around the mono- and multi-NP clusters of similar size but consisting of the mixture of NSPs and NRs. Standard (one excitation wavelength) and rainbow (several excitation wavelengths) mechanisms were quantitatively compared through the two PNB parameters (Table 1): the maximal size of PNB was characterized by their lifetime (Figure 2C), and the optical brightness of PNB was characterized through the pixel image amplitude (Figure 2A, B). The clusters that yielded similar scattering image amplitudes (at the level of 1060 counts) were studied. In addition, the composition and dimensions of the clusters were verified with scanning electron microscopy (SEM). According to their SEM images, the size of these clusters was 130-180 nm. Under identical excitation conditions (532 $\mathrm{nm}: 16 \mathrm{~mJ} / \mathrm{cm}^{2}, 787 \mathrm{~nm} 36 \mathrm{~mJ} / \mathrm{cm}^{2}$ ) the mono-clusters of NSPs and NRs returned no or small PNBs, while the multi-NP clusters of the same size returned relatively large PNBs when being exposed to the two simultaneous laser pulses of different wavelengths (Table 1).

The results obtained (Table 1) showed the definite priority of the novel "rainbow" mechanism over the standard excitation of a single plasmon resonance: the lifetime and brightness of rainbow PNBs increased by almost one order of magnitude compared to the identical excitation of the mono-NP clusters.

In addition, we varied the fluence of the single laser pulse in order to achieve the same lifetime of the PNB around a mono-NP cluster as was obtained for the rainbow PNB (Figure 3B). To achieve the level of the lifetime (maximal diameter) of the rainbow PNB as shown in Figure $3 \mathrm{~B}$ with a mono-NSP cluster of the same size and with a single pulse excitation, we had to increase the laser pulse fluence 4-5 fold from 16 to $73 \mathrm{~mJ} / \mathrm{cm}^{2}$. Furthermore, under fixed laser fluence we increased the size of the mono-NP cluster and achieved a PNB lifetime close to that of the rainbow PNB. A similar PNB lifetime was achieved with clusters

\begin{tabular}{|l|l|l|}
\hline Sample & Bubble lifetime, ns & $\begin{array}{l}\text { The pixel image } \\
\text { amplitudes, count }\end{array}$ \\
\hline Mono-NP clusters of gold spheres & $3 \pm 4$ & $990 \pm 15$ \\
\hline Mono-NP clusters of gold rods & $3 \pm 3$ & $1045 \pm 31$ \\
\hline $\begin{array}{l}\text { Multi-NP clusters consisting of } \\
\text { mixed spheres and rods }\end{array}$ & $63 \pm 4$ & $8860 \pm 321$ \\
\hline
\end{tabular}

Table 1: Parameters of plasmonic nanobubbles generated around NP clusters exposed to two simultaneous laser pulses $\left(0.5 \mathrm{~ns}\right.$ each, $532 \mathrm{~nm}: 16 \mathrm{~mJ} / \mathrm{cm}^{2}, 787$ $\mathrm{nm} 36 \mathrm{~mJ} / \mathrm{cm}^{2}$ ). that were 3-4 times bigger (Figure 3A). Therefore, the simultaneous excitation of two plasmon resonances with the two optical pulses resulted in an amplification of the photothermal efficacy of the multiNP cluster that exceeded the effect expected from a single pulse excitation with the fluence being equal to the sum of the fluences of the two above pulses. The summarized fluence of the two pulses $(16 \mathrm{~mJ} /$ $\mathrm{cm}^{2}$ and $36 \mathrm{~mJ} / \mathrm{cm}^{2}$ ) was $52 \mathrm{~mJ} / \mathrm{cm}^{2}$, a value that was close to the PNB generation threshold for mono-NP clusters (see above). The lifetimes of PNBs observed at this level of fluence under a single pulse excitation of the mono-NP clusters were lower than that for the rainbow PNB (Figure 3B). In another experiment, identical NSP-NS clusters were exposed to single $532 \mathrm{~nm}$ pulses, single $787 \mathrm{~nm}$ pulses and the two simultaneous pulses $532 \mathrm{~nm}$ and $787 \mathrm{~nm}$ under fixed fluences near the PNB generation thresholds. While single pulse wavelengths returned no or small PNBs, synergistic double-pulse (rainbow) excitation resulted in the stable generation of significant PNBs (Figure 3C). Therefore, the rainbow PNB mechanism clearly demonstrates a synergistic nature and is thus principally different from the mechanism based on the excitation of a single plasmon resonance. This provides an opportunity for improving the selectivity and efficacy of the generation of rainbow PNBs in biomedical applications.

All of the above results were obtained for the NPs mixed in 1:1 concentration. It is difficult to expect such precise mixing in cells under heterogeneous conditions. We varied the ratio NSP: NR from 1:1 to 10:1 in order to prepare the mixed clusters of heterogeneous origin. We measured the PNB lifetime around mixed NP clusters of similar diameter so only their content (NP concentration ratio) was a variable. We did not observe a significant variation in the PNB lifetime for the clusters prepared under the above range. A similar result was obtained for the mixed clusters of $60 \mathrm{~nm}$ NSPs and $110 \mathrm{~nm}$ silica-gold NSs. These results demonstrate the improved stability of rainbow PNBs compared to the PNBs generated around mono-NP clusters which were found to be very sensitive to the variation of NP concentration that influence the NP cluster size. The results obtained were next applied to study the effect of rainbow PNBs in living cells in vitro.

\section{Rainbow plasmonic nanobubbles in living cells}

The formation of NP clusters in living cells (unlike the above water model) involves several biological processes that are heterogeneous and cause rather uncontrollable variation of NP uptake, NP cluster size and thus reducing the selectivity of NP-based therapies. We have studied how the basic biological processes influence the selectivity and efficacy of PNB generation. In particular, we considered the following factors and processes:

1. The efficacy and specificity of initial NP targeting (that provides the initial accumulation of gold NPs at the cellular membrane) was supported by the combination of the two different antibodies that were covalently conjugated to specific gold NPs and bound to specific cell surface protein targets. We applied cell-specific (prostate cancer) gold conjugates of $60 \mathrm{~nm}$ NSP with PSMA (prostate specific membrane antigen) antibody and $110 \mathrm{~nm}$ gold NS conjugated with C225 (human EGF receptor) antibody that is tumor-associated, although it is less specific to prostate cancer cells than PSMA. The NRs were replaced by other NPs with NIR plasmon resonances, by NSs, because the latter turned out to be less toxic and easier to conjugate.

2. The clustering of the membrane-accumulated NPs was provided by their endocytosis and subsequent aggregation in endosomal compartments in the cytoplasm. This process depends upon the activity of the cellular physiological uptake systems. 


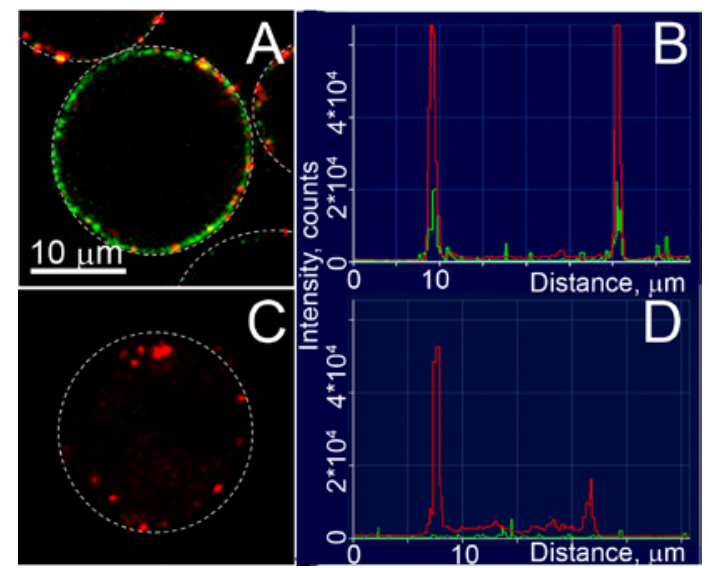

Figure 4: Merged confocal fluorescent (green) and scattering (red) images of prostate cancer C4-2B (A) and stromal HS-5 (C) cells: green - the fluorescence of Alexa Fluor 488 conjugated to PSMA antibody, red - the gold NP clusters; $(B, D)$ corresponding profiles of pixel image amplitudes. Dashed lines show the boundaries of cells.
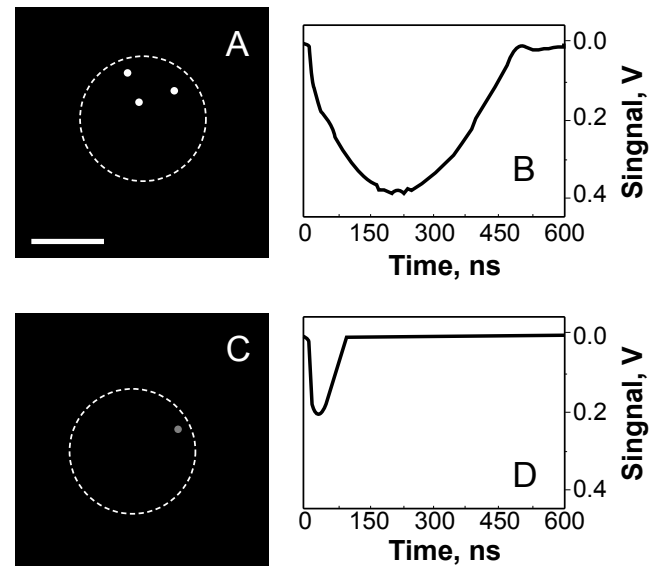

Figure 5: Time-resolved scattering images $(A, C)$ of the cells during their exposure to the simultaneous pair of laser pulses at $532 \mathrm{~nm}$ and $787 \mathrm{~nm}$ shows bright PNBs in C4-2B cell; (B,D): corresponding time responses obtained simultaneously with images $(A)$ and $(C)$. Scale bar is $10 \mu \mathrm{m}$. Dashed lines show the boundaries of cells.

The selectivity of rainbow PNBs was evaluated by identically treating specific (prostate cancer) target cells $\mathrm{C} 4-2 \mathrm{~B}$, and non-cancerous cells HS-5, typical of surrounding cancer stroma, through comparing the PNB parameters in both types of cell.

Initially we repeated the experiment described above for the NP clusters in water, and used receptor-mediated endocytosis instead of chemical methods for the formation of NP clusters in living cells. The cells were incubated with NSP-PSMA and NS-C225 conjugates separately and also with a 1:1 mixture of these conjugates. Accumulation of the gold NP conjugates in cells was imaged by using optical scattering mode of the laser scanning confocal microscope LSM-710 (Figure 4). Despite much higher level of specific receptors in target (C4-2B) cells (shown in green in Figure 4A) we observed significant level of nonspecific accumulation of gold NPs in stromal (control) cells (shown in red in Figure 4C). Profiles of scattering amplitude in Figures 4B, D gives the idea about the level of the accumulation of PSMA-specific receptors and gold NPs in both types of cells studied. The accumulation of gold NPs has been found to be rather heterogeneous in both types of cells. However, the size (scattering image pixel amplitude) of NP clusters in $\mathrm{C} 4-2 \mathrm{~B}$ cells has been found to be slightly bigger in prostate cancer cells (C4-2B) comparing to those in HS-5 cells. According to numerous studies and to our previous experimental experience, non-specific accumulation of NPs is unavoidable due to the residual level of interacting receptors at the membranes of non-specific cells and, in additional, depends on the cellular uptake activity. Observed heterogeneity of NP clustering in cells may decrease the specificity of many NP-based diagnostic and therapeutic methods including the standard mechanism of PNB generation.

Therefore, we have applied the standard and the rainbow mechanisms of PNB generation to individual living cells. Each C4$2 \mathrm{~B}$ cell was exposed to laser pulses at $532 \mathrm{~nm}, 787 \mathrm{~nm}$ and to the simultaneous pair of these pulses, each one having the fixed fluence of $95 \mathrm{~mJ} / \mathrm{cm}^{2}$. The PNBs were detected in time-resolved scattering and time-response modes (Figure 5). The PNB lifetimes and the probabilities of their generation were plotted as shown in the diagram (Figure 6A). The rainbow mechanism (targeting the cells with NSs and NSPs and exposing them to the two simultaneous laser pulses, $532 \mathrm{~nm}$ and $787 \mathrm{~nm}$ ) resulted in an increase in PNB size (lifetime) of almost one order of magnitude compared to the response of the same cells to mono-NP/mono-pulse treatments with (1) NSP-PSMA targeting and a single laser pulse $(532 \mathrm{~nm})$ or (2) NS-C225 targeting and a single laser pulse $(787 \mathrm{~nm})$. Thus the results obtained with the cell model, confirmed our previous conclusion, that the efficacy of the generation of rainbow PNB was much higher than for standard PNBs and represents synergistic effect of the dual pulse excitation of the two matching NP types in one cluster. It should be noted that we could not achieve the same parameters for PNBs (lifetime about $500 \mathrm{~ns}$ ) after targeting the cells only with gold NSPs and a single wavelength (532 $\mathrm{nm}$ ) excitation, because the required laser pulse fluence exceeded the technical maximum of the laser. According to the results obtained in water for NSP clusters, we assume that the fluence should be increased by 3-4 fold compared to the rainbow PNB fluence. Such a high efficacy of rainbow PNBs in cancer cells can be explained by the synergistic effect of the co-localized and simultaneous activation of the several plasmon resonances.

To verify these conclusions we measured the PNBs in C4-2B cells under lower fluences. Rainbow PNBs of 95 ns were achieved under the excitation 532nm@40mJ/ $\mathrm{cm}^{2} / 787 \mathrm{~nm} @ 48 \mathrm{~mJ} / \mathrm{cm}^{2}$ (the summarized fluence was $88 \mathrm{~mJ} / \mathrm{cm}^{2}$ ). We intentionally reduced the levels of laser fluences compared to the previous experiments so as to generate the smallest PNBs. Standard PNBs of 95 ns were achieved under the excitation 532nm@215mJ/ $\mathrm{cm}^{2}$, this fluence level exceeded the above summarized fluence of the two laser pulses by 2.4 times. This confirmed the synergistic effect of the co-localized excitation of several plasmon resonances.

Next, we have compared the PNBs in three different sets of the prostate cancer cells (C4-2B) prepared under the different initial ratios of the NSPs:NSs concentrations (aimed to influence the composition of the NP clusters) and treated with various laser pulses (one pulse $532 \mathrm{~nm}$ or $787 \mathrm{~nm}$ and two simultaneous pulses $532 \mathrm{~nm}$ and $787 \mathrm{~nm}$ ) under identical conditions $\left(55 \mathrm{~mJ} / \mathrm{cm}^{2}\right)$. The data in Figure $6 \mathrm{~B}$ show the minimal spread for the lifetimes of rainbow PNBs while the activation of similarly targeted cells with only one of the laser pulses resulted in much higher spread of the PNB lifetime. Therefore, the varying targeting concentrations ratios of gold NPs (that modeled the influence of biological heterogeneous factors) had minimal effect on the rainbow PNBs while they showed much stronger influence on the PNB parameters under a single pulse excitation of one type of gold NPs 
in the cells. This experiment has modeled the heterogeneous conditions during NP-cell interaction that influence the content of the NP clusters. However, synergistic targeting and activation of NP clusters with at least two laser pulses (rainbow PNBs) minimized the influence of the variation of the NP cluster content on the PNB lifetime and probability and, therefore, a rainbow PNB mechanism can be employed for the improved control of PNB generation in biologically heterogeneous microenvironment.

Finally, we evaluated the rainbow PNBs for the selective ablation of cancer cells surrounded by non-cancer stromal cells (a typical cancer microenvironment). We fluorescently labeled stromal (HS-5) and prostate cancer cells (C4-2B) with calcein vital dyes of green (cancer) and orange (stromal) colors and mixed them in the proportion HS5:C4-2B of 5:1. Next, we treated the mixture of cells with the two gold conjugates and with two laser pulses, $532 \mathrm{~nm} @ 40 \mathrm{~mJ} / \mathrm{cm}^{2}$ and 787 $\mathrm{nm} @ 48 \mathrm{~mJ} / \mathrm{cm}^{2}$. The sample was scanned through the laser beam and each cell was identified according to its fluorescence (Figure 7B), and was then exposed to a single pair of laser pulses (Figure 7C). Based upon the PNB generation probabilities and lifetimes (Figure 6A), the stable PNBs were generated under the rainbow mechanism and in cancer cells only. PNBs were also observed on occasion in stromal cells, but their probability was below $20 \%$ and their size was small compared to the size of cancer cell-generated PNBs. This latter result inspired an additional test: because cell damage by a PNB depends upon its size (lifetime [27, 37]), we monitored and compared the vital fluorescence of PNB treated cancerous and stromal cells.

Within the area irradiated with laser pulses (shown within a dashed line in Figure 7B, C) we discovered that only the cancer cell was damaged. The vital dye stays in the cell with an intact membrane and quickly leaks out of the cell with a compromised membrane [45]. The damage (ablation) of the cancer cell in the center (observed as the loss of the red fluorescence) was in line with the increased size (lifetime) of the PNBs generated specifically in C4-2B cells, and with the mechanical nature of cell damage that is associated with the disruption of the cellular structures including the plasma membrane [27,33,37,46,47]. This final test clearly demonstrated the selectivity of the rainbow PNBs that were generated locally in a single specific (cancer) cell and were not generated in the surrounding stromal cells (or were generated in such cells with a probability and size that prevented their damage). While the vital fluorescent images in Figure 7B, C demonstrated the potential of rainbow PNBs for selective therapy, the optical scattering of these PNBs has a high diagnostic potential.

The brightness and optical contrast of rainbow PNBs were compared to those of gold NPs (scattering). The brightness of the PNBs in cancer C4-2B cells (measured as the pixel image amplitude of the PNB, Figure $5 \mathrm{~A}$ ) was found to be 71 times higher than that for stromal cells (Figure 5C). The accumulation of the gold NP conjugates in cells was imaged by using optical scattering mode of the laser scanning confocal microscope (Figure 4). After measuring cluster-related image amplitudes we have estimated the contrast of NP optical scattering. It was measured as a ratio of the scattering image amplitudes for cancer and stromal cells and was found to be about 1.5. Significant increase in optical contrast due to the rainbow PNB was provided by the threshold mechanism of PNB generation: none or only small PNBs were generated in HS- 5 cells (Figure 5C, D) because the level of laser fluence was close to the PNB threshold for the smallest clusters formed in HS- 5 cells, while the same fluence level exceeded the PNB generation threshold for larger clusters formed in C4-2B cells (Figure 5A, B).

\section{Rainbow mechanism versus other methods of optical excitation of plasmonic NPs}

The rainbow PNB method further develops the unique properties of PNBs through the synergistic excitation of PNB around multi-NP cluster. There are several mechanisms for the optical activation of plasmonic NPs. The most popular, excitation, employs continuous radiation [50-52], which is a relatively simple and low cost solution. However, the continuous delivery of optical energy to an NP compromises the efficacy and spatial selectivity of the photothermal output of such an NP due to thermal diffusion [53]. This problem was solved by using short laser pulses $[11,12,54-56]$. Very short (fs) single pulses improve the photothermal efficacy by minimizing heat loss by NPs, though at the same time they limit the efficacy of PT conversion since a maximal energy is limited by the optical breakdown threshold. For this reason many fs-pulsed lasers are used in continuous mode
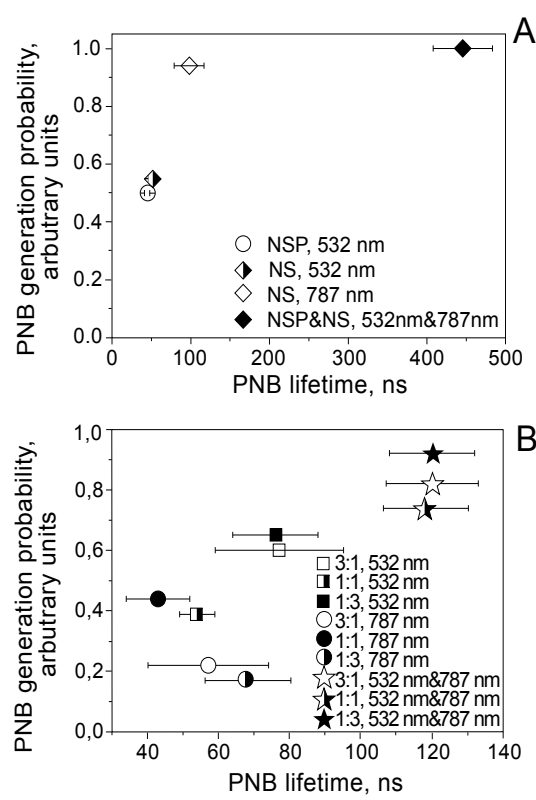

Figure 6: A: The PNB lifetime-probability diagram for prostate cancer (C4-2B) cells treated with NSP-PSMA and NS-C225 gold conjugates, and exposed to different optical pulses (pump laser pulses $0.5 \mathrm{~ns} @ 95 \mathrm{~mJ} / \mathrm{cm}^{2}$ at $532 \mathrm{~nm}$ and $787 \mathrm{~nm}$ ). The rainbow mechanism corresponds to the sample shown with a solid rhomb (cells were treated with the two conjugates and two simultaneous laser pulses); B: the PNB lifetime-probability diagram for prostate cance (C4-2B) cells shows the influence of the NP cluster content (the ratio of NSC225:NSP-PSMA) on the PNB parameters under excitation with one 0.5-ns laser pulse $532 \mathrm{~nm} @ 55 \mathrm{~mJ} / \mathrm{cm}^{2}$ or $787 \mathrm{~nm} @ 55 \mathrm{~mJ} / \mathrm{cm}^{2}$ (single wavelength excitation), and two simultaneous laser pulses - $532 \mathrm{~nm}$ and $787 \mathrm{~nm}$ (rainbow mechanism).

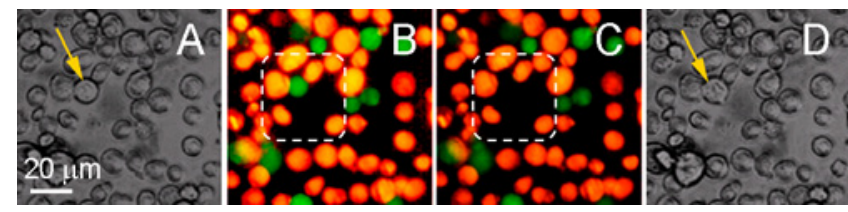

Figure 7: Bright field (A, D) and fluorescent (B, C: C4-2B - green, HS-5 - orange) images of co-culture of prostate cancer $\mathrm{C} 4-2 \mathrm{~B}$ and stromal HS-5 cells. (A, B): before exposure to the pair of laser pulses $(532 \mathrm{~nm}$ and $787 \mathrm{~nm}) ;(C, D): 60 \mathrm{~s}$ after exposure to the laser pulses that were scanning along the area shown with a dashed line. Fading of green fluorescence was due to the leakage of green calcein dye out of the damaged C4-2B cell through its disrupted membrane (shown with yellow arrow). Yellow arrows show a single cancer cell that has been selectively damage with the rainbow mechanism of PNB generation. 
$[26,57]$. Other than as thermal sources, plasmonic NPs are employed as sources for acoustic, shock waves and vapor bubbles. Among these mechanisms the gold nanoparticle-generated vapor bubbles (PNBs) were shown to provide the best selectivity and accuracy for cell-level biomedical applications [58]. Compared to a "standard" mechanism of nanobubble generation around a gold NP in a cell (antibodyreceptor targeting, activation of mono-NP cluster with a single optical wavelength), the rainbow PNB mechanism introduced several specific features:

1. Nanobubble source: clusters of different NPs, rather than clusters of NPs of one type. This feature decreased the bubble generation threshold fluence below the threshold level for mono-NP clusters.

2. Excitation optical wavelength and fluence: several simultaneous pulses at the wavelengths that match the plasmon resonances of the corresponding NPs in the cluster. This feature also reduced the fluence of each laser pulse below the threshold for a nanobubble generated through a "single pulse - mono-NP" mechanism.

3. Independent control and tuning of the fluence: each excitation laser pulse provided maximal flexibility in manipulating the lifetime (i.e. size) of the rainbow PNB in a wide range of cluster size and composition, thus circumventing the problem of the heterogeneous formation and content of NP clusters in cells.

All three above features are unique to rainbow PNBs and can be especially useful during in vivo applications where the heterogeneity of NP clustering and optical propagation and scattering create challenges both for nano- and for optical technologies. It should be noted that a rainbow PNB depends much less upon the variations of its NP content (this could be caused by the heterogeneous interaction and internalization of gold NPs by the cell). This result, together with the tunable and multifunctional properties of PNBs in vitro and in vivo $[27,33,47]$ that support diagnosis, therapy and theranostics (diagnosis and treatment united in one procedure) at cell level and with single cell selectivity, justify the development of PNB technologies despite the above general limitations.

We conclude that the method of "rainbow plasmonic nanobubbles" demonstrated the following advantages over the photothermal excitation of plasmonic NPs and PNBs in a single plasmon resonance mode:

1. The rainbow mechanism of the PNB generation significantly improved the efficacy of their generation by reducing the fluence of the excitation laser pulses by several times to the level that was below the PNB generation thresholds for single NPs and for clusters consisting of only one type of NPs.

2. The mechanism of rainbow plasmonic nanobubbles improved the selectivity of bubble generation in specific target cancer cells compared to non-specific cells and can therefore provide the cell level selectivity that is very important for efficient diagnosis and therapy.

3. Rainbow plasmonic nanobubbles were found to be less sensitive to the NP cluster content and can be better controlled through the fluence of the excitation laser pulses compared to plasmonic nanobubbles generated around mono-NP clusters whose content can vary in cells due to the heterogeneous nature of NP uptake by living cells.

Obtained results open the possibility for very precise and localized control of the photothermal excitation of plasmonic nanoparticles in heterogeneous micro-environment at the cell level.

\section{Acknowledgements}

This work was supported by the National Institute of Health, grants 1R21CA133641 and R01GM094816 to D. O. L. and E. Y. L. H., P01CA098912 to M. C. F. C. Authors thank Professor S. Link (Rice University) for providing gold nanorods, Dr. V. Nammalvar (Rice University) for providing gold nanoshells and the members of the Carson laboratory for many helpful discussions. Ms. Sue Parminter has kindly copy-edited the manuscript. Confocal microscopy was performed on equipment obtained through a Shared Instrumentation Grant S10RR026399-01 from the National Institutes of Health.

\section{References}

1. Hirsch LR, Gobin AM, Lowery AR, Tam F, Drezek RA et al. (2006) Metal nanoshells. Ann Biomed Eng 34: 15-22.

2. Murphy CJ, Gole AM, Stone JW, Sisco PN, Alkilany AM, et al. (2008) Gold nanoparticles in biology: beyond toxicity to cellular imaging. Acc Chem Res 41: $1721-1730$.

3. Javier D Nitin N, Roblyer D Richards-Kortum R (2008) Metal-based nanorods as molecule-specific contrast agents for reflectance imaging in $3 \mathrm{D}$ tissues. $J$ Nanophotonics 2: 023506.

4. Hu M, Chen J, Li Z, Au L, Hartland G, et al. (2006) Gold nanostructures: engineering their plasmonic properties for biomedical applications. Chem Soc Rev 35: 1084-1094.

5. Cherukuri P, Glazer ES, Curley SA (2010) Targeted hyperthermia using metal nanoparticles. Adv Drug Deliv Rev 62: 339-345.

6. Huang X, Jain PK, El-Sayed IH, El-Sayed MA (2008) Plasmonic photothermal therapy (PPTT) using gold nanoparticles. Lasers Med Sci 23: 217-228.

7. Farny H, Wu T, Holt R, Murray T, Roy R (2005) Nucleating cavitation from laserilluminated nano-particles. Acoust Res Lett Online 6: 138-143.

8. Kotaidis V, Dahmen C, von Plessen G, Springer F, Plech A (2006) Excitation of nanoscale vapor bubbles at the surface of gold nanoparticles in water. $J$ Chem Phys124: 184702.

9. Prasad V, Mikhailovsky A, Zasadzinski JA (2005) Inside-out disruption of silica/ gold core-shell nanoparticles by pulsed laser irradiation. Langmuir 21: 75287532.

10. Liu Z, Hung WH, Aykol M, Valley D, Cronin SB (2010) Optical manipulation of plasmonic nanoparticles, bubble formation and patterning of SERS aggregates. Nanotechnology 21: 105304

11. Wang S, Chen K-J, Wu T-H, Wang H, Lin W-Y, et al. (2010) Phototherma effects of supramolecularly assembled gold nanoparticles for the targeted treatment of cancer cells. Angew Chem Int Ed 49: 3777-3781.

12. Wu T-H, Kalim S, Callahan C, Teitell M, Chiou PY (2010) Image patterned molecular delivery into live cells using gold particle coated substrates. Opt Express 18: 938-946.

13. McLaughlan JR, Roy RA, Ju H, Murray TW (2010) Ultrasonic enhancement of photoacoustic emissions by nanoparticle-targeted cavitation. Opt Lett 35: 2127-2129.

14. Wang Y, Xie X, Wang X, Ku G, Gill KL (2004) Photoacoustic tomography of a nanoshell contrast agent in the in vivo rat brain. Nano Letters 4: 1689-1692.

15. Taylor U, Klein S, Petersen S, Kues W, Barcikowski S (2007) Nonendosomal cellular uptake of ligand-free, positively charged gold nanoparticles. Cytometry A 77: 439-446.

16. Oberdörster G, Elder A, Rinderknecht A (2009) Nanoparticles and the brain cause for concern? J Nanosci Nanotechnol 9: 4996-5007.

17. Lasagna-Reeves C, Gonzalez-Romero D, Barria MA, Olmedo I, Clos A, et al. (2010) Bioaccumulation and toxicity of gold nanoparticles after repeated administration in mice. Biochem Biophys Res Commun 393: 649-655.

18. Gu Y, Cheng J, Lin C, Lam Y, Cheng S, et al. (2009) Nuclear penetration of surface functionalized gold nanoparticles. Toxicol Appl Pharmacol 237: 196204

19. Kogan MJ, Olmedo I, Hosta L, Guerrero AR, Cruz LJ (2007) Peptides and metallic nanoparticles for biomedical applications. Nanomedicine 2: 287-306.

20. Li J, Wang L, Liu X, Zhang Z, Guo H, et al. (2009) In vitro cancer cell imaging and therapy using transferrin-conjugated gold nanoparticles. Cancer Lett 274 319-326. 
Citation: Lukianova-Hleb EY, Oginsky AO, Shenefelt DL, Drezek RA, Hafner JH, et al. (2011) Rainbow Plasmonic Nanobubbles: Synergistic Activation of Gold Nanoparticle Clusters. J Nanomedic Nanotechnol 2:104. doi:10.4172/2157-7439.1000104

21. Arnida, Malugin A, Ghandehari $\mathrm{H}$ (2009) Cellular uptake and toxicity of gold nanoparticles in prostate cancer cells: a comparative study of rods and spheres. J Appl Toxicol 30: 212-217.

22. Patra CR, Bhattacharya R, Mukhopadhyay D, Mukherjee P (2010) Fabrication of gold nanoparticles for targeted therapy in pancreatic cancer. Adv Drug Deliv Rev 62: 346-361.

23. Tong L, Wei Q, Wei A, Cheng JX (2009) Gold nanorods as contrast agents for biological imaging: optical properties, surface conjugation and photothermal effects. Photochem Photobiol 85: 21-32.

24. Huang X, El-Sayed IH, El-Sayed MA (2010) Applications of gold nanorods for cancer imaging and photothermal therapy. Methods Mol Biol 624: 343-57.

25. Chen J, Glaus C, Laforest R, Zhang Q, Yang M, et al. (2010) Gold nanocages as photothermal transducers for cancer treatment. Small 6: 811-817.

26. Tong L, Zhao Y, Huff T, Hansen M, Wei A. et al. (2007) Gold nanorods mediate tumor cell death by compromising membrane integrity. Adv. Mater. 19: 31363141.

27. Lukianova-Hleb E, Hanna E, Hafner J, Lapotko D (2010) Tunable plasmonic nanobubbles for cell theranostics. Nanotechnology 21: 085102.

28. Lapotko D, Lukianova-Hleb E, Oraevsky A (2007) Clusterization of nanoparticles during their interaction with living cells. Nanomedicine 2: 241-253.

29. Hleb EY, Hafner JH, Myers JN, Hanna EY, Rostro BC, et al. (2008) LANTCET elimination of solid tumor cells with photothermal bubbles generated around clusters of gold nanoparticles. Nanomedicine 3:647-667.

30. Lapotko D (2009) Optical excitation and detection of vapor bubbles around plasmonic nanoparticles. Opt Express 17: 2538-2556.

31. Lukianova-Hleb EY, Hu Y, Latterini L, Tarpani L, Lee S, et al. (2010) Plasmonic nanobubbles as transient vapor nanobubbles generated around plasmonic nanoparticles. ACS Nano 4: 2109-2123.

32. Lapotko D, Lukianova E, Oraevsky A (2006) Selective laser nano-thermolysis of human leukemia cells with microbubbles generated around clusters of gold nanoparticles. Lasers Surg Med 38: 631-642.

33. Wagner DS, Delk NA, Lukianova-Hleb EY, Hafner JH, Farach-Carson MC et al (2010) The in vivo performance of plasmonic nanobubbles as cell theranostic agents in zebrafish hosting prostate cancer xenografts. Biomaterials 31: 7567-

34. Brandenberger C, Mühlfeld C, Ali Z, Lenz AG, Schmid O, et al. (2010) Quantitative evaluation of cellular uptake and trafficking of plain and polyethylene glycol-coated gold nanoparticles. Small 6: 1669-1678.

35. Mailänder V, Landfester K (2009) Interaction of nanoparticles with cells. Biomacromolecules 10: 2379-2400.

36. Jiang W, Kim BY, Rutka JT, Chan WC (2008) Nanoparticle-mediated cellular response is size-dependent. Nat Nanotechnol 3: 145-150

37. Lapotko D, Lukianova K, Shnip A (2005) Photothermal responses of individual cells. J Biomed Optics 10: 014006.

38. Silver DA, Pellicer I, Fair WR, Heston WD, Cordon-Cardo C (1997) Prostatespecific membrane antigen expression in and malignant human tissues. Clin. Cancer Res. 3: 81-85.

39. Wright GL, Haley C, Beckett ML, Schellhammer PF (1995) Expression of prostate-specific membrane antigen in normal, benign, and malignant prostate tissues. Ural. Oncol 1:18-28.

40. Denmeade SR, Sokoll LJ, Dalrymple S, Rosen DM, Gady AM, et al. (2003)
Dissociation between androgen responsiveness for malignant growth vs. expression of prostate specific differentiation markers PSA, hK2, and PSMA in human prostate cancer models. Prostate 54: 249-257.

41. Grandis JR, Sok JC (2004) Signaling through the epidermal growth facto receptor during the development of malignancy. Pharmacol. Ther. 102: 37-46.

42. O'Connor JC, Farach-Carson MC, Schneider CJ, Carson DD (2007) Coculture with prostate cancer cells alters endoglin expression and attenuates transforming growth factor-beta signaling in reactive bone marrow stroma cells. Mol. Cancer Res. 5: 585-603.

43. Liu H, Rajasekaran AK, Moy P, Xia Y, Kim S et al. (1998) Constitutive and antibody-induced internalization of prostate-specific membrane antigen. Cancer Res. 58: 4055-4060.

44. Lapotko D, Lukianova-Hleb E, OraevskyA(2007) Clusterization of nanoparticles during their interaction with living cells. Nanomedicine 2: 241-253.

45. Mueller H, Kassack MU, Wiese M (2004) Comparison of the usefulness of the MTT, ATP, and calcein assays to predict the potency of cytotoxic agents in various human cancer cell lines. J Biomol Screen 9: 506-515.

46. Lapotko D, Romanovskaya T, Gordiyko E (2002) Photothermal monitoring of redox state of respiratory chain in single live cells. Photochem Photobiol 75 519-526.

47. Lukianova-Hleb E, Santiago C, Wagner D, Hafner J, Lapotko D (2010) Generation and detection of plasmonic nanobubbles in zebrafish. Nanotechnology 21: 225102

48. Hleb E, Lapotko D (2009) Influence of transient environmental phototherma effects on optical scattering by gold nanoparticles. Nano Letters 9: 2160-2166

49. Hleb E, Hu Y, Drezek R, Hafner J, Lapotko D (2008) Photothermal bubbles as optical scattering probes for imaging living cells. Nanomedicine 3: 797-812.

50. Stern JM, Stanfield J, Kabbani W, Hsieh JT, Cadeddu JA (2008) Selective prostate cancer thermal ablation with laser activated gold nanoshells. J Uro 179: $748-753$

51. Huang HC, Rege K, Heys JJ (2010) Spatiotemporal temperature distribution and cancer cell death in response to extracellular hyperthermia induced by gold nanorods. ACS Nano 4: 2892-2900.

52. Govorov O, Richardson H (2007) Generating heat with metal nanoparticles Nano Today 1:30-38

53. Anderson RR, Parrish JA (1983) Selective photothermolysis: precise microsurgery by selective absorption of pulsed radiation. Science 220: 524 527

54. Pitsillides M, Joe EK, Wei X, Anderson RR, Lin CP (2003) Selective cell targeting with light-absorbing microparticles and nanoparticles. Biophys $\mathrm{J} 84$ : 4023-4032.

55. Green DE, Longtin JP, Sitharaman B (2009) The effect of nanoparticleenhanced photoacoustic stimulation on multipotent marrow stromal cells. ACS Nano 32065-2072.

56. Volkov AN, Sevilla C, Zhigilei LV (2007) Numerical modeling of short pulse lase interaction with au nanoparticle surrounded by water. Appl Surf Sci 253: 6394 6399

57. Huang X, Qian W, El-Sayed IH, El-Sayed MA (2007) The potential use of the enhanced nonlinear properties of gold nanospheres in photothermal cance therapy. Lasers Surg. Med. 39: 747-753.

58. Lapotko D (2009) Plasmonic nanoparticle-generated photothermal bubbles and their biomedical applications. Nanomedicine 7:813-845. 